\title{
LOWER BOUND FOR THE HIGHER MOMENT OF SYMMETRIC SQUARE $L$-FUNCTIONS
}

\author{
GUANGHUA JI
}

\begin{abstract}
Let $\mathcal{S}_{k}(N)$ be the space of holomorphic cusp forms of weight $k$, level $N$ and let $\mathcal{B}_{k}(N)$ be an orthogonal basis of $\mathcal{S}_{k}(N)$ consisting of newforms. Let $L\left(s, \operatorname{sym}^{2} f\right)$ be the symmetric square $L$-function of $f \in$ $\mathcal{B}_{k}(N)$. In this paper, the lower bound of the higher moment of $L\left(1 / 2, \operatorname{sym}^{2} f\right)$ is established, i.e., for any even positive number $r$,
\end{abstract}

$$
\sum_{f \in \mathcal{B}_{k}(N)} \omega_{f}^{-1} L\left(\frac{1}{2}, \operatorname{sym}^{2} f\right)^{r} \gg(\log N)^{r(r+1) / 2}
$$

holds for $N \rightarrow \infty$.

1. Introduction and statement of results. An important problem in number theory is to determine the asymptotic formula of the moments of central values of $L$-functions varying in a family. This problem has been intensively studied in recent years.

Katz and Sarnak [7] have introduced the idea of a family of $L$ functions with an associated symmetry type, and they gave strong evidence that the symmetry group governs many properties of the distribution of zeros of the $L$-functions. Later, Conrey and Farmer [3] proved that the symmetry group also governs the behavior of the mean values of the $L$-functions.

The general conjecture for the moment of $L$-functions can be obtained by random matrix theory. For a family $\mathcal{F}$ of $L$-functions with functional equations $s \mapsto 1-s$ and appropriate gamma factors,

2010 AMS Mathematics subject classification. Primary 11F11, 11F66.

Keywords and phrases. Holomorphic forms, lower bound, symmetric square $L$ functions.

The author is partially supported by the young scholars program of Shandong University, NSF of Shandong Province and NSF (grant No. 11301299).

Received by the editors on May 2, 2014, and in revised form on July 23, 2014. 
$L(1 / 2, f)$ is the central value. The conjecture is that, as $D \rightarrow \infty$,

$$
\frac{1}{D^{*}} \sum_{\substack{f \in \mathcal{F} \\ c(f) \leq D}} L\left(\frac{1}{2}, f\right)^{r} \sim \frac{a_{r} g_{r}}{\Gamma(1+B(r))}\left(\log D^{A}\right)^{B(r)},
$$

where the family $\mathcal{F}$ is partially ordered by the conductor $c(f)$, and $D^{*}$ the number of elements with $c(f) \leq D$, for details, see [3]. Let $L\left(s, \operatorname{sym}^{2} f\right)$ be the symmetric square $L$-function of $f \in \mathcal{B}_{k}(N)$. In the context of the moment of $L\left(1 / 2, \operatorname{sym}^{2} f\right)$, random matrix theory says that one should have, as $N \rightarrow \infty$,

$$
\sum_{f \in \mathcal{B}_{k}(N)} \omega_{f}^{-1} L\left(\frac{1}{2}, \operatorname{sym}^{2} f\right)^{r} \sim C(k, r)(\log N)^{r(r+1) / 2} .
$$

The above asymptotic formulas for $r=1,2$ are known, see [2].

Following the idea of Rudnick and Soundararajan [11], we shall consider the lower bounds for the moments of symmetric square $L$ functions in this paper. Our main theorem is as follows.

Theorem 1.1. Let $k$ be a fixed even integer and $N$ a squarefree number. Let $L\left(s, \operatorname{sym}^{2} f\right)$ be the symmetric square $L$-function of $f \in \mathcal{B}_{k}(N)$. For any even positive number $r$, we have

$$
\sum_{f \in \mathcal{B}_{k}(N)} \omega_{f}^{-1} L\left(\frac{1}{2}, \operatorname{sym}^{2} f\right)^{r} \gg(\log N)^{r(r+1) / 2}
$$

holds for $N \rightarrow \infty$.

The research on lower bounds for the moments of the central values of $L$-functions began with Ramachandra [9] and Heath-Brown [4], and has been studied by many authors, see $[1,6,8,10,11,12]$. In particular, Rudnick and Soundararajan [11] and Tang [12] obtained the lower bound of the moments of $L(s, f)$ and $L\left(s, \operatorname{sym}^{2} f\right)$ in the weight $k$ aspect for the full modular group $S L(2, \mathbb{Z})$, respectively. And in the paper, [8], Radziwill and Soundararajan extended the idea of Rudnick and Soundararajan to obtain lower bounds of the Riemann zeta function for all real numbers $r \geq 1$. Following the method of Rudnick and Soundararajan, we can obtain lower bounds for these moments in Theorem 1.1 for all rational numbers $r \geq 1$. 


\section{Preliminaries.}

2.1. Holomorphic cusp forms. For a fixed even positive integer $k$ and a squarefree number $N$, the space $\mathcal{S}_{k}(N)$ of the holomorphic cusp forms of the weight $k$ for the Hecke congruence subgroup $\Gamma_{0}(N)$ is a finite-dimensional Hilbert space with respect to the Petersson inner product

$$
\langle f, g\rangle=\int_{\Gamma_{0}(N) \backslash \mathbb{H}} f(z) \overline{g(z)} y^{k-2} \mathrm{~d} x \mathrm{~d} y, \quad f, g \in \mathcal{S}_{k}(N) .
$$

Let $\mathcal{B}_{k}(N)$ be an orthogonal basis of $\mathcal{S}_{k}(N)$ consisting of newforms, that is, normalized eigenforms for all Hecke operators. We write the Fourier expansion of a newform $f \in \mathcal{B}_{k}(N)$

$$
f(z)=\sum_{n \geq 1} \lambda_{f}(n) n^{(k-1) / 2} e(n z) .
$$

Then the Ramanujan conjecture, now a theorem due to Deligen, says

$$
\left|\lambda_{f}(n)\right| \leq \tau(n),
$$

where $\tau(n)$ is the number of divisors of $n$. The Hecke eigenvalues $\lambda_{f}(n)$ satisfy the relation

$$
\lambda_{f}(1)=1, \quad \lambda_{f}(m) \lambda_{f}(n)=\sum_{d \mid(m, n)} \lambda_{f}\left(\frac{m n}{d^{2}}\right) .
$$

2.2. Peterssson trace formula. Write the weight function

$$
\omega_{f}=\frac{(4 \pi)^{k-1}}{\Gamma(k-1)}\langle f, f\rangle=\frac{(k-1) N}{2 \pi^{2}} L\left(1, \operatorname{sym}^{2} f\right),
$$

where $\langle f, f\rangle$ is the Petersson inner product and $L\left(s, \operatorname{sym}^{2} f\right)$ is the symmetric square $L$-function of $f(z)$. The following bound is well known

$$
\frac{1}{N \log ^{3} N} \ll \omega_{f}^{-1} \ll \frac{\log N}{N} .
$$

The Petersson trace formula states that

$$
\sum_{f \in \mathcal{B}_{k}(N)} \omega_{f}^{-1} \lambda_{f}(m) \lambda_{f}(n)=\delta(m, n)+2 \pi i^{-k} \sum_{N \mid c} \frac{S(m, n ; c)}{c} J_{k-1}\left(\frac{4 \pi \sqrt{m n}}{c}\right),
$$


where $\delta(m, n)$ is the diagonal symbol, $J_{k-1}(x)$ is the standard $J$-Bessel function and $S(m, n ; c)$ is the classical Kloosterman sum defined by

$$
S(m, n ; c)=\sum_{d \bar{d} \equiv 1(\bmod c)} e\left(\frac{m d+n \bar{d}}{c}\right) .
$$

Using the estimate $J_{k-1}(x) \ll \min (1, x / k)$ and the Weil bound for Kloosterman sums, we have the following result, see [5, Corollary 14.24].

Lemma 2.1. With notation as above, we have for any $m, n \geq 1$,

$$
\begin{aligned}
& \sum_{f \in \mathcal{B}_{k}(N)} \omega_{f}^{-1} \lambda_{f}(m) \lambda_{f}(n) \\
= & \delta(m, n)+O\left(\tau_{3}((m, n))(m, n, N)^{1 / 2}(m n)^{1 / 4} \frac{\tau(N)}{N \sqrt{k}} \log \left(1+\frac{(m n)^{1 / 4}}{\sqrt{N k}}\right)\right),
\end{aligned}
$$

where the implied constant is absolute.

2.3. Symmetric square $L$-functions. For a newform $f \in \mathcal{B}_{k}(N)$, the symmetric square $L$-function is given by

$$
L\left(s, \operatorname{sym}^{2} f\right)=\zeta^{(N)}(2 s) \sum_{n \geq 1} \frac{\lambda_{f}\left(n^{2}\right)}{n^{s}},
$$

where $\zeta^{(N)}(s)$ is the Riemann zeta function with the local factor at the prime $N$ removed. The completed $L$-function $\Lambda\left(s, \operatorname{sym}^{2} f\right)=$ $N^{s} L_{\infty}\left(s, \operatorname{sym}^{2} f\right) L\left(s, \operatorname{sym}^{2} f\right)$ is entire and it satisfies the functional equation

$$
\Lambda\left(s, \operatorname{sym}^{2} f\right)=\Lambda\left(1-s, \operatorname{sym}^{2} f\right),
$$

where

$$
L_{\infty}\left(s, \operatorname{sym}^{2} f\right)=\pi^{-3 s / 2} \Gamma\left(\frac{s+1}{2}\right) \Gamma\left(\frac{s+k+1}{2}\right) \Gamma\left(\frac{s+k}{2}\right),
$$

is the local factor at the infinity place. The first moment of central values of the symmetric square $L$-functions is established, see [2]. 
Lemma 2.2. For $(m, N)=1$ and any $\epsilon>0$, one has

$$
\begin{aligned}
& \sum_{f \in \mathcal{B}_{k}(N)} \omega_{f}^{-1} L\left(\frac{1}{2}, \operatorname{sym}^{2} f\right) \lambda_{f}\left(m^{2}\right) \\
& =\frac{1}{\sqrt{m}}\left(2 \gamma+\frac{L_{\infty}^{\prime}}{L_{\infty}}\left(\frac{1}{2}, \operatorname{sym}^{2} f\right)+\log \frac{\sqrt{N}}{m}+\sum_{p \mid N} \frac{2 \log p}{p-1}\right) \\
& +O_{k, \epsilon}\left(\left(\frac{m}{N^{3 / 4}}+\frac{1}{N^{1 / 4}}\right)(m N)^{\epsilon}\right) .
\end{aligned}
$$

\section{Proof of the theorem.}

Proof. Let $x=N^{1 / 2 r}$ and consider

$$
A(f)=A(f, x)=\sum_{n \leq x} \frac{\lambda_{f}\left(n^{2}\right)}{\sqrt{n}} .
$$

Define

$$
S_{1}=\sum_{f \in \mathcal{B}_{k}(N)} \omega_{f}^{-1} L\left(\frac{1}{2}, \operatorname{sym}^{2} f\right) A(f)^{r-1},
$$

and

$$
S_{2}=\sum_{f \in \mathcal{B}_{k}(N)} \omega_{f}^{-1} A(f)^{r},
$$

where $r$ is an even positive integer. By Hölder's inequality, we have

$$
\begin{aligned}
& \left(\sum_{f \in \mathcal{B}_{k}(N)} \omega_{f}^{-1} L\left(\frac{1}{2}, \operatorname{sym}^{2} f\right) A(f)^{r-1}\right)^{r} \\
& \quad \leq\left(\sum_{f \in \mathcal{B}_{k}(N)} \omega_{f}^{-1} L\left(\frac{1}{2}, \operatorname{sym}^{2} f\right)^{r}\right)\left(\sum_{f \in \mathcal{B}_{k}(N)} \omega_{f}^{-1} A(f)^{r}\right)^{r-1},
\end{aligned}
$$

which gives

$$
\sum_{f \in \mathcal{B}_{k}(N)} \omega_{f}^{-1} L\left(\frac{1}{2}, \operatorname{sym}^{2} f\right)^{r} \geq \frac{S_{1}^{r}}{S_{2}^{r-1}} .
$$


We will prove the main theorem by finding the asymptotic orders of the magnitude of $S_{1}$ and $S_{2}$.

In order to get the asymptotic formula of $S_{2}$, we need to study the combinatorics of the Fourier coefficient $\lambda_{f}\left(n^{2}\right)$. Following from the Hecke multiplicity (2.1), we may write

$$
\lambda_{f}\left(n_{1}^{2}\right) \lambda_{f}\left(n_{2}^{2}\right) \cdots \lambda_{f}\left(n_{r}^{2}\right)=\sum_{t \mid n_{1} n_{2} \cdots n_{r}} b_{t}\left(n_{1}, n_{2}, \ldots, n_{r}\right) \lambda_{f}\left(t^{2}\right),
$$

where $b_{t}\left(n_{1}, n_{2}, \ldots, n_{r}\right)$ are certain non-negative integers which satisfy

$$
b_{t}\left(n_{1}, n_{2}, \ldots, n_{r}\right) \ll\left(n_{1} n_{2} \cdots n_{r}\right)^{\epsilon} .
$$

It is easy to see that $b_{1}$ satisfies a multiplicative property. If

$$
\left(\prod_{i=1}^{r} m_{i}, \prod_{i=1}^{r} n_{i}\right)=1
$$

then

$$
b_{1}\left(m_{1} n_{1}, m_{2} n_{2}, \ldots, m_{r} n_{r}\right)=b_{1}\left(m_{1}, m_{2}, \ldots, m_{r}\right) b_{1}\left(n_{1}, n_{2}, \ldots, n_{r}\right) .
$$

Denote

$$
B_{r}(n)=\sum_{\substack{n_{1}, n_{2}, \ldots, n_{r} \\ n_{1} n_{2} \cdots n_{r}=n}} b_{1}\left(n_{1}, n_{2}, \ldots, n_{r}\right)
$$

then we have that $B_{r}(n)$ is a multiplicative function. Note that $B_{r}\left(p^{a}\right)$ is independent of $p$ and grows at most polynomially in $a$ and

$$
B_{r}(p)=0, B_{r}\left(p^{2}\right)=\frac{r(r-1)}{2},
$$

which follows from

$$
b_{1}(p, 1, \ldots, 1)=b_{1}\left(p^{2}, 1, \ldots, 1\right)=0
$$

and

$$
b_{1}(p, p, \ldots, 1)=1 \text {. }
$$

Consequently, we can estimate $S_{2}$ as follows:

$$
S_{2}=\sum_{n_{1}, n_{2}, \ldots, n_{r} \leq x} \frac{1}{\sqrt{n_{1} n_{2} \cdots n_{r}}}
$$




$$
\begin{aligned}
& \sum_{t \mid n_{1} n_{2} \cdots n_{r}} b_{t}\left(n_{1}, n_{2}, \ldots, n_{r}\right) \sum_{f \in \mathcal{B}_{k}(N)} \omega_{f}^{-1} \lambda_{f}\left(t^{2}\right) \\
= & \sum_{n_{1}, n_{2}, \ldots, n_{r} \leq x} \frac{b_{1}\left(n_{1}, n_{2}, \ldots, n_{r}\right)}{\sqrt{n_{1} n_{2} \cdots n_{r}}} \\
& +O\left(N^{-3 / 2+\epsilon} x^{r} \sum_{n_{1}, n_{2}, \ldots, n_{r} \leq x} \frac{1}{\left(n_{1} n_{2} \cdots n_{r}\right)^{1 / 2-\epsilon}}\right) \\
= & \sum_{n_{1}, n_{2}, \ldots, n_{r} \leq x} \frac{b_{1}\left(n_{1}, n_{2}, \ldots, n_{r}\right)}{\sqrt{n_{1} n_{2} \cdots n_{r}}} \\
& +O\left(N^{-3 / 4+\epsilon}\right),
\end{aligned}
$$

where we have used Lemma 2.1 in the second step. As for the main term, we have

$$
\sum_{n \leq x} \frac{B_{r}(n)}{\sqrt{n}} \leq \sum_{n_{1}, n_{2}, \ldots, n_{r} \leq x} \frac{b_{1}\left(n_{1}, n_{2}, \ldots, n_{r}\right)}{\sqrt{n_{1} n_{2} \cdots n_{r}}} \leq \sum_{n \leq x^{r}} \frac{B_{r}(n)}{\sqrt{n}}
$$

By the properties of $B_{r}(n)$, we have that the generating function satisfies

$$
\sum_{n=1}^{\infty} \frac{B_{r}(n)}{n^{s}}=\zeta(2 s)^{r(r-1) / 2} D(s),
$$

where $D(s)$ is a Dirichlet series which converges absolutely in $\Re s>$ $1 / 3+\epsilon$. A standard argument gives that, for a positive constant $C_{r}$,

$$
\sum_{n \leq z} \frac{B_{r}(n)}{\sqrt{n}} \sim C_{r}(\log z)^{r(r-1) / 2}
$$

Finally, we get

$$
S_{2} \asymp(\log x)^{r(r-1) / 2} \asymp(\log N)^{r(r-1) / 2} .
$$

Returning to $S_{1}$, note that

$$
A(f)^{r-1}=\sum_{n_{1}, \ldots, n_{r-1} \leq x} \frac{1}{\sqrt{n_{1} \cdots n_{r-1}}} \sum_{t \mid n_{1} \cdots n_{r-1}} b_{t}\left(n_{1}, \ldots, n_{r-1}\right) \lambda_{f}\left(t^{2}\right) .
$$


By bound (2.2), we obtain

$$
\begin{aligned}
S_{1}= & \sum_{n_{1}, n_{2}, \ldots, n_{r-1} \leq x} \frac{\sum_{t \mid n_{1} n_{2} \cdots n_{r-1}} b_{t}\left(n_{1}, n_{2}, \ldots, n_{r-1}\right)}{\sqrt{n_{1} n_{2} \cdots n_{r-1}}} \\
& \sum_{f \in \mathcal{B}_{k}(N)} \omega_{f}^{-1} L\left(\frac{1}{2}, \operatorname{sym}^{2} f\right) \lambda_{f}\left(t^{2}\right) \\
= & \frac{1}{2} \log N \sum_{n_{1}, n_{2}, \ldots, n_{r-1} \leq x} \frac{1}{\sqrt{n_{1} n_{2} \cdots n_{r-1}}} \\
& \sum_{t \mid n_{1} n_{2} \cdots n_{r-1}} \frac{b_{t}\left(n_{1}, n_{2}, \ldots, n_{r-1}\right)}{\sqrt{t}}+O\left(N^{-1 / 4 r+\epsilon}\right) .
\end{aligned}
$$

Note that

$$
b_{1}\left(n_{1}, n_{2}, \ldots, n_{r-1}, t\right)= \begin{cases}b_{t}\left(n_{1}, n_{2}, \ldots, n_{r-1}\right) & \text { if } t \mid n_{1} n_{2} \cdots n_{r-1}, \\ 0 & \text { otherwise }\end{cases}
$$

Therefore,

$$
S_{1}=\frac{1}{2} \log N \sum_{\substack{n_{1}, n_{2}, \ldots, n_{r-1} \leq x \\ n_{r} \leq x^{r-1}}} \frac{b_{1}\left(n_{1}, n_{2}, \ldots, n_{r-1}, n_{r}\right)}{\sqrt{n_{1} n_{2} \cdots n_{r-1} n_{r}}}+O\left(N^{-1 / 4 r+\epsilon}\right) .
$$

Using $b_{1}\left(n_{1}, n_{2}, \ldots, n_{r-1}, n_{r}\right) \geq 0$, we have that $S_{1} \gg(\log N) S_{2}$. Arguing, as in the case of $S_{2}$, we finally get that

$$
S_{1} \gg(\log N)^{1+r(r-1) / 2} \text {. }
$$

From (3.1) and (3.2), we have

$$
\frac{S_{1}^{r}}{S_{2}^{r-1}} \gg(\log N)^{r(r+1) / 2},
$$

where the implied constant depends on $r$. This completes the proof.

Acknowledgments. The author would like to thank the Department of Mathematics at the Ohio State University for its hospitality during his visit in 2014. He would also like to thank the referee for valuable suggestions and detailed comments. 


\section{REFERENCES}

1. A. Akbary and B. Fodden, Lower bounds for power moments of L-functions, Acta Arith. 151 (2012), 11-38.

2. V. Blomer, On the central value of symmetric square L-functions, Math. Z. 260 (2008), 755-777.

3. J.B Conrey and D.W Farmer, Mean values of L-functions and symmetry, Int. Math. Res. Not. 2000 (2000), 883-908.

4. D.R Heath-Brown, Fractional moments of the Riemann zeta function, J. Lond. Math. Soc. 24 (1981), 65-78.

5. H. Iwaniec and E. Kowalski, Analytic number theorem, Amer. Math. Soc. Colloq. Publ. 53, American Mathematical Society, Providence, 2004.

6. G.H. Ji, Lower bounds for moments of automorphic L-functions over short intervals, Proc. Amer. Math. Soc. 137 (2009), 3569-3574.

7. N.M. Katz and P. Sarnak, Zeroes of zeta functions and symmetry, Bull. Amer. Math. Soc. 36 (1999), 1-26.

8. M. Radziwill and K. Soundararajan, Continuous lower bounds for moments of zeta and L-functions, Mathematika 59 (2013), 119-128.

9. K. Ramachandra, Some remarks on the mean value of the Riemann zetafunction and other Dirichlet series, I, Hardy-Ramanujan J. 1 (1978), 1-15.

10. Z. Rudnick and K. Soundararajan, Lower bounds for moments of L-functions, Proc. Natl. Acad. Sci. 102 (2005), 6837-6838.

11.

Lower bounds for moments of L-functions: Symplectic and orthogonal examples, in Multiple Dirichlet series, automorphic forms, and analytic number theory, Proc. Sympos. Pure Math. 75, American Mathematical Society, Providence, RI, 2006.

12. H.C. Tang, Lower bound for the higher moment of symmetric square $L$ functions, J. Numer. Theor. 133 (2013), 2143-2152.

School of Mathematics, Shandong University, Jinan, Shandong 250100, P.R. CHINA

Email address: ghji@sdu.edu.cn 